\title{
Correction to: Relevance of biomarkers across different neurodegenerative diseases
}

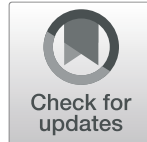

\author{
Alexander J. Ehrenberg ${ }^{1,2,3^{*}}$, Ayesha Khatun ${ }^{4}$, Emma Coomans ${ }^{5}$, Matthew J. Betts ${ }^{6,7}$, Federica Capraro ${ }^{8,9}$, \\ Elisabeth H. Thijssen ${ }^{1,10}$, Konstantin Senkevich ${ }^{11,12}$, Tehmina Bharucha ${ }^{13}$, Mehrsa Jafarpour ${ }^{14}$, Peter N. E. Young ${ }^{15,16}$, \\ William Jagust ${ }^{3,17}$, Stephen F. Carter ${ }^{18,19}$, Tammaryn Lashley ${ }^{14,20}$, Lea T. Grinberg ${ }^{1,21,22}$, Joana B. Pereira ${ }^{23,24}$, \\ Niklas Mattsson-Carlgren ${ }^{15,16}$, Nicholas J. Ashton ${ }^{15,16,25,26}$, Jörg Hanrieder ${ }^{14,15}$, Henrik Zetterberg ${ }^{14,15,27,28}$, \\ Michael Schöll ${ }^{14,15,24}$ and Ross W. Paterson ${ }^{4}$
}

\section{Correction to: Alz Res Ther 12, 56 (2020) https://doi.org/10.1186/s13195-020-00601-w}

Following publication of the original article [1], the authors reported a production error in the article title. The last word "diseases" was omitted by copy editor.

The original article [1] has been updated.

\begin{abstract}
Author details
${ }^{1}$ Memory and Aging Center, Weill Institute for Neurosciences, University of California, San Francisco, San Francisco, CA, USA. ${ }^{2}$ Department of Integrative Biology, University of California, Berkeley, Berkeley, CA, USA. ${ }^{3}$ Helen Wills Neuroscience Institute, University of California, Berkeley, Berkeley, CA, USA. ${ }^{4}$ Dementia Research Centre, University College London Institute of Neurology, London, UK. ${ }^{5}$ Department of Radiology \& Nuclear Medicine, Amsterdam UMC, location VUmc, Amsterdam Neuroscience, Amsterdam, The Netherlands. ${ }^{6}$ German Center for Neurodegenerative Diseases (DZNE), Magdeburg, Germany. ${ }^{7}$ Institute of Cognitive Neurology and Dementia Research, Otto von Guericke University Magdeburg, Magdeburg, Germany. ${ }^{8}$ The Francis Crick Institute, London, UK. ${ }^{9}$ Department of Neuromuscular Diseases, University College London Queen Square Institute of Neurology, London, UK. ${ }^{10}$ Department of Clinical Chemistry, Amsterdam UMC, Amsterdam, The Netherlands. "'Petersburg Nuclear Physics Institute names by B.P. Konstantinov of National Research Center, Kurchatov Institute, St. Petersburg, Russia. ${ }^{12}$ First Pavlov State Medical University of St. Petersburg, St. Petersburg, Russia. ${ }^{13}$ Oxford Glycobiology Institute, Department of Biochemistry, University of Oxford, Oxford, UK. ${ }^{14}$ Department of Neurodegenerative Disease, UCL Queen Square, Institute of Neurology, University College London, London, UK. ${ }^{15}$ Department of Psychiatry and Neurochemistry, Sahlgrenska Academy at the University of Gothenburg,
\end{abstract}

Mölndal, Sweden. ${ }^{16}$ Wallenberg Center for Molecular and Translational Medicine, Lund University, Lund, Sweden. ${ }^{17}$ Molecular Biophysics and Integrated Bioimaging, Lawrence Berkeley National Laboratory, Berkeley, CA, USA. ${ }^{18}$ Department of Psychiatry, School of Clinical Medicine, University of Cambridge, Cambridge, UK. ${ }^{19}$ Wolfson Molecular Imaging Centre, Division of Neuroscience and Experimental Psychology, University of Manchester, Manchester, UK. ${ }^{20}$ Queen Square Brain Bank for Neurological Disorders, UCL Queen Square Institute of Neurology, London, UK. ${ }^{21}$ University of São Paulo Medical School, São Paulo, Brazil. ${ }^{22}$ Global Brain Health Institute, San Francisco, CA, USA. ${ }^{23}$ Division of Clinical Geriatrics, Department of Neurobiology, Care Sciences and Society, Karolinska Institutet, Stockholm, Sweden. ${ }^{24}$ Clinical Memory Research Unit, Department of Clinical Sciences, Faculty of Medicine, Lund University, Lund, Sweden. ${ }^{25}$ King's College London, Institute of Psychiatry, Psychology \& Neuroscience, Maurice Wohl Clinical Neuroscience Institute, London, UK. ${ }^{26} \mathrm{NIHR}$ Biomedical Research Centre for Mental Health \& Biomedical Research Unit for Dementia at South London \& Maudsley NHS Foundation, London, UK. ${ }^{27}$ Clinical Neurochemistry Laboratory, Sahlgrenska University Hospital, Mölndal, Sweden. ${ }^{28}$ UK Dementia Research Institute at University College London, London, UK.

Published online: 09 June 2020

\section{Reference}

1. Ehrenberg, A.J., Khatun, A., Coomans, E. et al. Relevance of biomarkers across different neurodegenerative diseases. Alz Res Ther. 2020;12:56. https://doi.org/10.1186/s13195-020-00601-w.

The original article can be found online at https://doi.org/10.1186/s13195020-00601-w.

* Correspondence: alexander.ehrenberg@ucsf.edu

${ }^{1}$ Memory and Aging Center, Weill Institute for Neurosciences, University of California, San Francisco, San Francisco, CA, USA

${ }^{2}$ Department of Integrative Biology, University of California, Berkeley, Berkeley, CA, USA

Full list of author information is available at the end of the article

(c) The Author(s). 2020 Open Access This article is licensed under a Creative Commons Attribution 4.0 International License, which permits use, sharing, adaptation, distribution and reproduction in any medium or format, as long as you give appropriate credit to the original author(s) and the source, provide a link to the Creative Commons licence, and indicate if changes were made. The images or other third party material in this article are included in the article's Creative Commons licence, unless indicated otherwise in a credit line to the material. If material is not included in the article's Creative Commons licence and your intended use is not permitted by statutory regulation or exceeds the permitted use, you will need to obtain permission directly from the copyright holder. To view a copy of this licence, visit http://creativecommons.org/licenses/by/4.0/ The Creative Commons Public Domain Dedication waiver (http://creativecommons.org/publicdomain/zero/1.0/) applies to the data made available in this article, unless otherwise stated in a credit line to the data. 